\title{
The impacts of some environmental pollutants and exogenous toxic ligands on conformational and functional properties of human hemoglobin: A systematic review of spectroscopic literature
}

\author{
Elmira Rezaei Pajouhesh ${ }^{1 \mathbb{D}}$, Ali Khatibi ${ }^{1 * \mathbb{(})}$ \\ ${ }^{1}$ Department of Biotechnology, Faculty of Biological Sciences, Alzahra University, Tehran, Iran
}

*Corresponding Author: Ali Khatibi, Department of Biotechnology, Faculty of Biological Sciences, Alzahra University, Sheikh Bahai Square, Tehran, Iran. Tel: +98-9124970501, Email: khatibi@alzahra.ac.ir

\begin{abstract}
Background and aims: The objective of the present study was to collect the spectroscopic results obtained from previous studies to determine how exogenous toxic ligands can alter the structural and functional properties of human hemoglobin $(\mathrm{HHb})$.

Methods: Using spectroscopic techniques, English published articles (from January 2009 to June 2020) on HHb were systematical reviewed by searching several databases such as PubMed, ScienceDirect databases, and Google Scholar. In this regard, some keywords were searched, including (human hemoglobin) AND (spectroscopy) OR (spectroscopic analysis) OR (spectroscopic studies) AND (environmental pollutants) OR (food additives) OR (dyes) OR (fuel additives) OR (radioactive metal ions) OR (insecticides) OR (food colorants). After screening full text articles, we extracted relevant data according to our subject from included articles.

Results: Finally, 17 studies were found to be related to the subject of this study. Analysis of investigations suggested that after exposure of $\mathrm{HHb}$ to each exogenous ligand, we could observe heme hydrophobic pocket alteration, fluorescence quenching, and polarity alteration around aromatic residues of protein, indicating that the structural properties of $\mathrm{HHb}$ were changing. Lack of some spectroscopic techniques in some articles could place some minor limitations on the obtained evidence.

Conclusion: Considering the impacts of the exogenous ligands on characteristics of $\mathrm{HHb}$, in the first step, it is the responsibility of governments to exercise strict control over the manufacturing sectors and then industry owners to investigate the effects of chemical products on the living organisms before commercializing them.

Keywords: Human hemoglobin, Environmental pollutant, Ultraviolet-visible absorbance, Circular dichroism
\end{abstract}

Received: 20 June 2021, Accepted: 18 September 2021, ePublished: December 62021

\section{Introduction}

The bulk of hemoglobin ( $\mathrm{Hb})$ in adults is found to be in the form of hemoglobin A ( $\mathrm{HbA})$, which plays a vital role in the bloodstream. This most abundant respiratory blood protein can physiologically act as an oxygen and carbon dioxide carrier, blood $\mathrm{pH}$ regulator, and hydrogen peroxide scavenger $(1,2)$. Further, it can be involved in transportation of proton and electron to all body organs and transportation of 2, 3-bisphosphogelycerate from tissues to kidneys $(3,4)$. Despite endogenous physiological conditions, $\mathrm{Hb}$ can be influenced by a variety of exogenous agents and environmental pollutants $(5,6)$. Although some physiochemical properties of these agents turn them into the environment threats for human health, their increasing use along with the development of societies and human needs is considered as a serious issue worldwide. For example, high water solubility of some insecticides and fuel additives accompanied by their poor soil adsorption provides them with a high potential to contaminate the surface and ground water sources
(5,7-9). This occurrence directly or indirectly influences the health of living creatures, specially humans, leading to a wide range of disorders in them.

There are several ways for these agents to penetrate to the human's body, including oral, dermal, and inhalation routes or through wound or contact with eyes. Except for the exposure way, these materials eventually enter the bloodstream through other ways. If they are capable of binding to blood proteins such as $\mathrm{Hb}$, this complexation not also will affect their distribution, metabolism, and cell uptake but also will lead to the alteration in protein folding and structural changes, subsequently causing functional failure in the macromolecule $(5,10)$. As such, the aim of the present review was to investigate the impacts of some environmental pollutants and exogenous toxic ligands on the conformational and functional properties of human hemoglobin $(\mathrm{HHb})$ through spectroscopic analysis.

\section{Materials and Methods}

Based on the study objective, the intended studies, which

(C) 2021 The Author(s); Published by Shahrekord University of Medical Sciences. This is an open-access article distributed under the terms of the Creative Commons Attribution License (http://creativecommons.org/licenses/by/4.0), which permits unrestricted use, distribution, and reproduction in any medium, provided the original work is properly cited. 
were published in English on $\mathrm{HHb}$ and were performed on environmental pollutants and exogenous toxic ligands, were reviewed using spectroscopic techniques. We ignored the studies which investigated the effects of drugs and nanoparticles on $\mathrm{HHb}$. We carried out systematic literature searches using the PubMed and ScienceDirect databases and google scholar search from January 2009 to June 2020 to identify spectroscopic studies, which were performed to investigate the impact of some environmental pollutants on $\mathrm{HHb}$ structure. In our advanced searches, we only selected articles from the relevant journals by applying filters provided in each database and then limited the types of journals according to our intended subjects. Afterwards, searching process was performed by using the following terms: human hemoglobin, spectroscopy, spectroscopic analysis, spectroscopic studies, environmental pollutants, food additives, dyes, fuel additives, radioactive metal ions, insecticides, food colorants. The search included all published English articles containing $\mathrm{HHb}$ and spectroscopic analysis in their studies. Primarily, the topic and abstract of each article were examined by the reviewer. In some cases that the topic was not clear, we needed to check out the article content. After the first step screening, some articles were rejected and were included in the list of exclusion criteria which were as follows: 1) studies which did not provide sufficient information to complete our investigation, 2) studies with double-publication analysis, 3) unrelated studies in terms of the article content, and 4) studies which were performed on drugs and nanoparticles. Mendeley software was considered useful as a referencing machine for data collection. During the screening process, we also reviewed the bibliography of the chosen articles to select the relevant papers, which have been probably missed during the PubMed and ScienceDirect searches. In this study, despite collecting spectroscopic data such as circular dichroism (CD), Ultravioletvisible (UV-vis), as well as normal and synchronous fluorescence spectroscopy, we tried to collect information on complementary variables (e.g., thermodynamic parameters, Stern-Volmer parameters, binding constant, and the number of binding sites), which provide a deeper insight into how different exogenous ligands interact with $\mathrm{HHb}$ and disrupt its characteristics. Figure 1 displays an overview of searching plan.

Results

A total of 283 articles were obtained from PubMed and ScienceDirect searches. Finally, 17 articles were selected after meeting the assessment inclusion criteria set by the researchers. Seven studies were performed on dyes, two studies described the impacts of fuel additives, one study was conducted on para-quinone. In addition, two studies were devoted to insecticides and the effects of some exogenous factors such as radioactive metal ions, gamma ray, and mobile phone radiofrequency, while glucose and bisphenol A were investigated in separate articles. These articles are classified in Figure 1.

\section{Dyes \\ Malachite green}

The results of a study (3) indicated that fluorescence emission of $\mathrm{HHb}$ at $325 \mathrm{~nm}$ diminished regularly and was red-shifted to $330 \mathrm{~nm}$ in the presence of malachite green (MG). Temperature dependent fluorimetry and the well-known Stern-Volmer equation were used To elucidate the binding mechanism of MG to $\mathrm{Hb}$. There was an inverse correlation between $\mathrm{K}_{\mathrm{sv}}$ (the Stern-Volmer dynamic quenching constant) and $\mathrm{K}_{\mathrm{q}}$ (the quenching rate

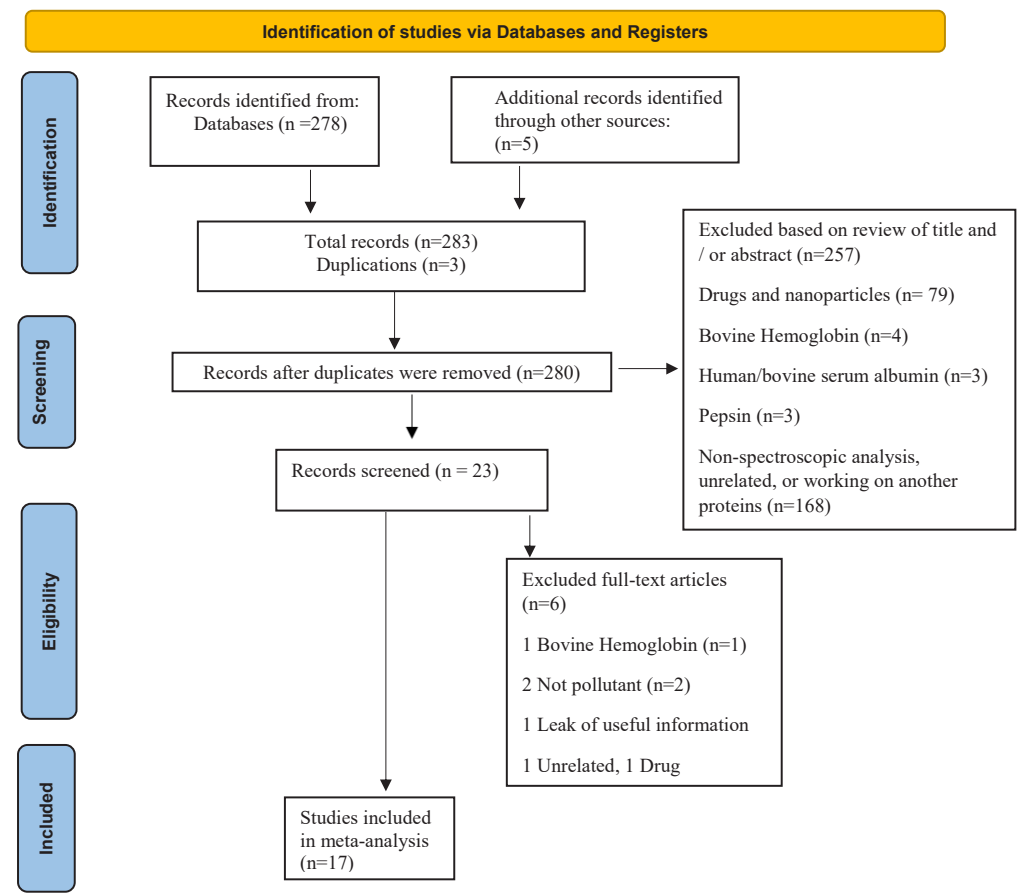

Figure 1. Article selection procedure. 
constant of the biomolecule) values with temperature. The values of binding constant $\left(\mathrm{K}_{\mathrm{b}}\right)$ decreased at higher temperatures, and the number of binding sites were found to be equal to one. Far-ultraviolet-circular dichroism (farUV CD) spectrum of $\mathrm{Hb}$ in the presence of dye exhibited a significant decrease at 208 and $222 \mathrm{~nm}$. Upon addition of MG, bathochromic effect at $\Delta \lambda=60 \mathrm{~nm}$ could be observed in the form of a slight red shift from $277 \mathrm{~nm}$ to 282.2 nm. All thermodynamic parameters such as $\Delta \mathrm{G}^{\circ}, \Delta \mathrm{H}^{\circ}$, and $\Delta \mathrm{S}^{\circ}$ showed negative signs. Extrinsic fluorescence intensity demonstrated that MG had potential to reduce 8- anilinonaphthalene-1- sulfonic acid (ANS) signal.

\section{Janus Green Blue}

A study (8) demonstrated that in the presence of Janus Green Blue (JGB), Hb absorption significantly decreased in both globin and Soret (or B) bands, and $2 \mathrm{~nm}$ blue shift was observed at globin peak maxima. More studies elucidated that $\mathrm{Hb}$ could potentially diminish the JGB absorbance at $600 \mathrm{~nm}$ and made it shift to blue about 8 $\mathrm{nm}$. The values of binding affinity were calculated to be approximately $8.6 \times 10^{4} \mathrm{M}^{-1}$.

Upon addition of JGB, a gradual decrease was observed in fluorescence intensity of tryptophan (Trp) residue at $328 \mathrm{~nm}$, while the position of the maxima remained unaltered. The values of $\mathrm{K}_{\mathrm{sv}}$ exhibited significant decrease through rising temperature. Further, the values of the number of binding sites ( $\mathrm{n}$ ) were around one. The analysis of quenching data demonstrated that $\mathrm{K}_{\mathrm{BL}}$ (the static quenching constant) decreased with raising temperature, which has conformity to the inverse dependency of $\mathrm{K}_{\mathrm{sv}}$ to temperature and static quenching mechanism. Addition of different concentration of JGB reduced the synchronous fluorescence intensity of $\mathrm{Hb}$. The peak maxima at $8 \mathrm{~nm}$ and $6 \mathrm{~nm}$ shifted to red and blue when the D-values (delta wavelengths) were set at 15 and $60 \mathrm{~nm}$, respectively. The effect of JGB on far-UV CD spectrum of $\mathrm{Hb}$ indicated a significant reduction of intensity at 208 and $222 \mathrm{~nm}$. From melting experiments, the $\mathrm{Hb}$ temperature melting point (Tm) was calculated to be about $59.5^{\circ} \mathrm{C}$, which reduced to $8^{\circ} \mathrm{C}$ upon addition of JGB.

Amaranth

Results of a study (11) demonstrated that upon addition of incremental amount of amaranth, $\mathrm{Hb}$ absorption spectrum displayed nearly $14 \%$ hypochromicity at 406 $\mathrm{nm}$ (characteristic for heme prosthetic group) while the position of the peak remained unchanged. After excitation at $295 \mathrm{~nm}, \mathrm{Hb}$ exhibited a strong fluorescence emission at $328 \mathrm{~nm}$, which decreased in the presence of amaranth. Moreover, the $\mathrm{K}_{\mathrm{sv}}$ parameter displayed a significant decrease along with rising temperature. Furthermore, in the presence of amaranth, the fluorescence intensity at $\Delta \lambda=60 \mathrm{~nm}$ decreased with a significant red shift while there was no shift in the tyrosine (Tyr) maximum spectrum at $\Delta \lambda=15 \mathrm{~nm}$. Based on the extrinsic fluorescence intensity results, addition of amaranth did not influence the $\mathrm{Hb}$ ANS complex. More studies demonstrated that amaranth can reduce $\mathrm{Hb}$ CD signal at 209 and $222 \mathrm{~nm}$.

Carmoisine

The obtained results from one study (12) indicated that the UV-vis absorption of $\mathrm{Hb}$, upon addition of carmoisine, exhibited around 9\% hypochromic change in the Soret bond (at $405 \mathrm{~nm}$ ) without any significant shift. The binding constant for $\mathrm{Hb}$-carmoisine complexation was estimated to be $6.45 \times 10^{4} \mathrm{M}^{-1}$. Regarding intrinsic fluorescence spectroscopy results, it is remarkable that carmoisine and $\mathrm{Hb}$ are both able to quench the emission of each other. The values of $\mathrm{K}_{\mathrm{sv}}$ and $\mathrm{K}_{\mathrm{q}}$ decreased with increasing temperature. More studies demonstrated that, as temperature rises, the binding constant decreases. Further, the values of the number of binding sites found to be equal to one. Synchronous fluorescence result at $\Delta \lambda=60 \mathrm{~nm}$ displayed the quenching of $\mathrm{Hb}$ intensity and noticeable red shift in the presence of carmoisine, while the position of peak at $\Delta \lambda=15 \mathrm{~nm}$ remained unaltered. The far-UV CD results demonstrated that upon addition of carmoisine, the CD signal reduced at both bands of 208 and $222 \mathrm{~nm}$.

\section{Tartrazine}

The obtained results from one study (13) demonstrated that by increasing the concentration of tartrazine (TZ), the $\mathrm{Hb}$ absorption peak at $406 \mathrm{~nm}$ displayed a hypochromicity about $6 \%$ while the position of the peak remained unaltered. The association constant for such complexation was calculated to be approximately $1.07 \times 10^{5} \mathrm{M}^{-1}$. Further, TZ was able to quench $\mathrm{Hb}$ emission spectra. Considering the data obtained from temperature dependent fluorimetry, it could be concluded that the both $\mathrm{K}_{\mathrm{sv}}$ and $\mathrm{K}_{\mathrm{q}}$ decreased with an increase in temperature. The values of binding constant and the number of binding sites were calculated to be $1.16 \times 10^{5} \mathrm{M}^{-1}$ and 1 , respectively. Furthermore, decrement of the binding constant, along with rising temperature could be observed. According to the synchronous fluorescence results, addition of TZ caused a marked decrease along with 6 and $3 \mathrm{~nm}$ red shift at $\Delta \lambda=60 \mathrm{~nm}$ and $\Delta \lambda=15 \mathrm{~nm}$ which were associated with $\operatorname{Trp}$ and Tyr residues, respectively. Upon addition of TZ, $\mathrm{Hb}$ CD signal decreased about $47 \%$ at two negative bonds of 210 and $222 \mathrm{~nm}$.

\section{Acid Red 27}

According to a study (14), it was found that Acid Red 27 (AR 27) caused $40 \%$ hypochromicity at $218 \mathrm{~nm}$ along with $8 \mathrm{~nm}$ blue shift; meanwhile, the absorbance of Soret band at $406 \mathrm{~nm}$ remained unaltered. This azo dye could also induce a regular reduction in $\mathrm{Hb}$ fluorescence, which was accompanied by a significant shift from 426 to 443 nm. More studies demonstrated that the Stern-Volmer plots for this quenching were linear and the maximum scatter collision quenching constant $\left(\mathrm{K}_{\mathrm{q}, \mathrm{r}}\right)$ was estimated to be about $2 \times 10^{10}$ L.mol/L.s which was less than the $\mathrm{K}_{\mathrm{q}}$ parameter with the order of $10^{12}$ L.mol/L.s. In addition, the values of $\mathrm{K}_{\mathrm{sv}}$ decreased with increasing temperature. 
Further analysis indicated that the number of binding sites for such interaction was equal to one, which decreased along with binding constant as the temperature increased. The signs of each thermodynamic parameter were $\Delta \mathrm{G}^{\circ}<0$, $\Delta \mathrm{H}^{\circ}<0$ and $\Delta \mathrm{S}^{\circ}>0$. The far-UV CD spectrum of $\mathrm{Hb}$ was influenced by AR 27, exhibiting a noticeable decrease at both negative bonds of 208 and $222 \mathrm{~nm}$. Upon addition of AR 27, a decrement in the synchronous fluorescence of $\mathrm{Hb}$ was observed at both $\Delta \lambda=15$ and $\Delta \lambda=60 \mathrm{~nm}$ which belonged to Tyr and Trp residues, respectively, and these quenching were accompanied by red shift in the emission maximum of both residues.

\section{Orange I and II}

In one study (15), the results indicated that addition of orange I and II to $\mathrm{Hb}$ solution led to an obvious decrease at 218 and $408 \mathrm{~nm}$. Fluorescence quenching of $\mathrm{Hb}$ was observed, and the analysis demonstrated that the rate constants for quenching process were greater than $2 \times 10^{10}$ L/mol.s. According to the obtained data, the values of binding constant did not show any obvious decrease with rising temperature. Further, the values of the number of binding sites were found to be equal to one. In addition, the signs of thermodynamic parameters were shown to be $\Delta \mathrm{G}^{\circ}<0, \Delta \mathrm{H}^{\circ}<0$ and $\Delta \mathrm{S}^{\circ}>0$. Moreover, decrement of the $\mathrm{Hb}$ ellipticity upon addition of dyes was observed in the far-UV CD analysis. From synchronous fluorescence study, it can be concluded that the dyes did not induce any significant changes in D-values of Tyr and Trp residues.

\section{Insecticides}

Imidacloprid

According to the results of a study (16), imidacloprid had the potential to reduce the $\mathrm{CD}$ signal of $\mathrm{Hb}$ at 208 and $222 \mathrm{~nm}$. It could also quench the intrinsic and extrinsic fluorescence intensity of $\mathrm{Hb}$ and $\mathrm{Hb}$-ANS. The values of $\mathrm{K}_{\mathrm{sv}}$ suggested an inverse correlation with temperature (exactly similar to the values of association constant), and the values of $\mathrm{K}_{\mathrm{q}}$ were considerably much greater than $\mathrm{K}_{\mathrm{sv}}$. More investigations indicated that the number of binding sites for imidacloprid on $\mathrm{Hb}$ was equal to one, and the values of thermodynamic parameters were fixed at negative signs.

Cartap

The obtained results from one study (17) revealed that upon addition of cartap, $\mathrm{Hb}$ absorbance increased at 280 $\mathrm{nm}$ along with a significant shift towards the red region, while the absorption diminished remarkably at both Soret and $\mathrm{Q}$ bands. The number of binding sites for cartap on protein was equal to two, and the sign of $\Delta \mathrm{G}$ for interaction of insecticide with globin region was more negative than that of protoporphyrin ring. It should be noted that, the $\mathrm{Hb}$ binding constant in the presence of cartap decreased with increasing temperature. Fluorimetry results displayed that after excitation of $\mathrm{Hb}$ at $460 \mathrm{~nm}$ in the presence of cartap, the emission peak displayed a clear growth at 460-550 $\mathrm{nm}$. Thermal denaturation study results at the wavelength of $280 \mathrm{~nm}$ indicated that increasing concentration of cartap led to the reduction of the protein Tm. Due to the turbidity measurement at $365 \mathrm{~nm}$, the $\mathrm{Hb}$ aggregation rate and the lag time increased and decreased, respectively, in the presence of cartap.

\section{Fuel additives}

Benzene

The obtained results from one study (5) indicated that the absorbance of $\mathrm{Hb}$ in the presence of benzene displayed hypochromicity in the Soret and Q bands (oxygen binding site) while the globin region showed a significant hyperchromicity at $275 \mathrm{~nm}$ with a clear red shift. After excitation at $321 \mathrm{~nm}$, the emission maxima at $460 \mathrm{~nm}$ enhanced due to the increase in the concentration of benzene. Significant increase in the slope of the logarithmic phase was observed along with increment of benzene concentration. In addition, dislocation of the protein $\mathrm{Tm}$ towards lower temperatures was found in the presence of benzene. Furthermore, upon addition of benzene, the peaks at $\Delta \lambda=17 \mathrm{~nm}$ and $\Delta \lambda=80 \mathrm{~nm}$ represented hypochromicity with an approximate blue shift.

\section{Methyl tertiary butyl ether}

The findings procured from one study (18) demonstrated that the thermal aggregation study of normal $\mathrm{HHb}$ at the wavelength of $360 \mathrm{~nm}$ showed a shorter lag phase in the presence of methyl tertiary butyl ether (MTBE). Furthermore, data obtained from the far-UV CD spectroscopy displayed a significant reduction in $\mathrm{Hb}$ CD signal. Further, an increase could be observed in the fluorescence intensity after protein excitation at 460 and $320 \mathrm{~nm}$ in the presence of MTBE.

\section{Other exogenous ligands Mobile phone radiofrequency}

One study (19) demonstrated that exposure of $\mathrm{Hb}$ to 940 $\mathrm{MHz}$ and $77 \mathrm{~V} / \mathrm{m}$ of electromagnetic field (EMF) and electric field intensity(EFI), respectively, caused a moderate increase in the far-UV CD signal of that protein. Despite exhibiting a clear reduction, fluorescence emission of the exposed $\mathrm{Hb}$, shifted to blue at $4 \mathrm{~nm}$. Meanwhile, extrinsic fluorescence of $\mathrm{Hb}$, which was generated from ANS probe, reduced when exposed to EMFs compared to the unexposed $\mathrm{Hb}$. The results obtained from the aggregation study indicated that exposing of $\mathrm{Hb}$ to $940 \mathrm{MHz}$ of EMFs and $77 \mathrm{~V} / \mathrm{m}$ of EFIs resulted in the protein aggregation and faster reach of protein to a plateau as compared with the unexposed protein. Further, the exposed $\mathrm{Hb}$ displayed $2^{\circ} \mathrm{C}$ increase at its $\mathrm{Tm}$ point compared with the unexposed $\mathrm{Hb}$.

\section{Lanthanides and actinides (La and Ac ions)}

Another study (20) found that increasing concentration of thorium (Th) (IV) from 10 to $100 \mu \mathrm{M}$ enhanced $\mathrm{Hb}$ 
absorbance at 211 and $277 \mathrm{~nm}$, which belonged to protein polypeptide chain and aromatic residues, respectively. Increasing effect of this metal ion on Soret band was observed in concentrations beyond $75 \mu \mathrm{M}$. The effect of uranium (U) (VI) from 10 to $75 \mu \mathrm{M}$ on $\mathrm{Hb}$ absorbance displayed a regular increase at 211 and $277 \mathrm{~nm}$, while at the concentrations more than $75 \mu \mathrm{M}$, the peak reached a saturation at $211 \mathrm{~nm}$ and shifted in baseline. At the concentrations more than $100 \mu \mathrm{M}$, the peak significantly increased at $406 \mathrm{~nm}$. To gain further information, the effect of lanthanides (La)s. on $\mathrm{Hb}$ was investigated, and the results indicated that both lanthanum (La) (III) and cerium (Ce) (III) within the concentration range of 10$100 \mu \mathrm{M}$ increased the absorbance at 211 and $277 \mathrm{~nm}$, while the absorbance peak remained unaffected at 406 $\mathrm{nm}$. In the following, the fluorimetry results revealed that upon addition of Th (IV), the fluorescence intensity of $\mathrm{Hb}$ decreased. In such case, the value of $\mathrm{K}_{\mathrm{sv}}$ and $\mathrm{K}_{\mathrm{q}}$, decreased with raising temperature from 298 to $310 \mathrm{~K}$, while those values increased in the case of $U(V I)$. Both values of the binding constant and the number of binding sites for Th (IV) were higher than that of U (VI). It should be noted that increasing temperature resulted in a decrease in binding constant for both Th (IV) and U (VI). Spectroscopic farUV CD analysis demonstrated a decrease in the CD signal of $\mathrm{Hb}$ at 209 and $222 \mathrm{~nm}$ in the presence of Th (IV) and $\mathrm{U}(\mathrm{VI})$, respectively, while their concentrations increased from 10 to $100 \mu \mathrm{M}$. It should be mentioned that Th (IV) at 75 and $100 \mu \mathrm{M}$ displayed a similar decrease in $\mathrm{Hb} \mathrm{CD}$ signal. In the case of Ce (III) and Ce (IV), the results suggested that 10 to $100 \mu \mathrm{M}$ of Ce (III) could significantly decrease CD signal, but no change was observed at 10 $\mu \mathrm{M}$ of $\mathrm{Ce}$ (IV). More investigations revealed that Ce (IV) induced intense alteration in the CD spectra of $\mathrm{Hb}$.

\section{Gamma ray}

One study (21) demonstrated that, upon $\gamma$-irradiation from 20 to 170 gray (Gy), the $\mathrm{Hb}$ absorption significantly decreased at all parts of the absorbance spectra (e.g., globin peak, Soret bond, and Q band). hen this reduction occurred, the positions of both globin and Q bands were fixed, but the Soret band and the peak at $348 \mathrm{~nm}$, which belong to Fe-His (iron- histidine) bond, were moved to blue and red regions, respectively. Analysis of the Q bands showed that concentration of the oxygen binding form of $\mathrm{Hb}$ (oxy- $\mathrm{Hb}$ ) decreased from 0 to $180 \mathrm{~Gy}$ in contrast to met and deoxy forms. $\gamma$-ray at doses higher than 170 Gy caused 15\% hyperchromicity in fluorescence intensity of $\mathrm{Hb}$ and made the peak more expanded. Increment of intensity at $278 \mathrm{~nm}$ was observed during synchronous fluorescence study, while D-value was fixed at $60 \mathrm{~nm}$ accompanied by generation of a new peak at $375 \mathrm{~nm}$. Heme degradation analysis showed that 170 Gy of $\gamma$-ray raised the fluorescence intensity at $465 \mathrm{~nm} 3$ times more than lower doses when excitation wavelength was fixed at $321 \mathrm{~nm}$. That is why the peak at $552 \mathrm{~nm}$ manifested a significant hyperchromicity just by 90 Gy of $\gamma$-ray after excitation at $460 \mathrm{~nm}$. More analysis showed that higher dose of $\gamma$-ray, which was chosen to be $170 \mathrm{~Gy}$ in the study, potentially decreased the protein's $\alpha$-helical content about $3 \%$. Furthermore, reduction of the Tm point could be clearly observed.

\section{Glucose}

The results of a study (22) indicated that incremental concentration of glucose (GLC), induces a significant decrease in the Trp absorption band $(280 \mathrm{~nm})$, the Soret region $(406 \mathrm{~nm})$, as well as Trp emission band (335 nm) after excitation at $280 \mathrm{~nm}$. In addition, GLC could increase far-Uv CD signals of $\mathrm{Hb}$.

\section{Bisphenol A 3,4-quinone}

One study (23) showed that the UV-Vis absorbance of $\mathrm{Hb}$ at 283 and $411 \mathrm{~nm}$ displayed a significant increase and a blue shift to 274 and $408 \mathrm{~nm}$ upon the addition of bisphenol A 3,4-quinone (BPAQ), respectively. Excitation of $\mathrm{Hb}$ at $280 \mathrm{~nm}$ in the presence of BPAQ resulted in regular decrement in the fluorescence emission of protein at $346 \mathrm{~nm}$ and a noticeable blue shift from 346 to $330 \mathrm{~nm}$. Further, the effect of BPAQ on Hb secondary structure displayed a reduction in CD signal in both negative peaks of 209 and $222 \mathrm{~nm}$.

\section{Para-quinone (P-benzoquinone)}

One study (24) has revealed that in the presence of P-benzoquinone, $16.6 \%$ reduction of the UV-vis absorption spectrum could be observed in smokers $\mathrm{Hb}$ at the wavelength of $406 \mathrm{~nm}$ (Soret bond) and decrement of the CD signal at 208 and $222 \mathrm{~nm}$. The impacts of all mentioned ligands on $\mathrm{HHb}$ spectral properties are collected in Table 1.

\section{Discussion}

The present study reviewed 17 articles on the effects of environmental pollutants on $\mathrm{HHb}$ Two of these studies investigated the impacts of fuel additives: MTBE and benzene. Interaction of MTBE with $\mathrm{HHb}$ induced reactive oxygen species (ROS) generation, which in turn increased protein aggregation. However, in the case of benzene, increase of hydrophobic surfaces on $\mathrm{HHb}$ - via protein unfolding led to protein aggregation. In both cases, the thermal aggregation curve displayed a short lag phase, suggesting that the rate of protein aggregation increased in the vicinity of MTBE and benzene (25). Existence of some secondary structures such as $\alpha$-helices in proteins offers them a significant stability. The loss of protein helicity reduced protein stability and increased the content of random coils, leading to protein aggregation (11). These phenomena were observed along with addition of MTBE and imidacloprid to $\mathrm{HHb}$ or its exposure to $170 \mathrm{~Gy}$ of $\gamma$-ray.

Heme, as prosthetic group of $\mathrm{Hb}$, is responsible for appearance of the Q bands in protein UV-vis spectra. Degradation of the prosthetic group reduced the intensity 


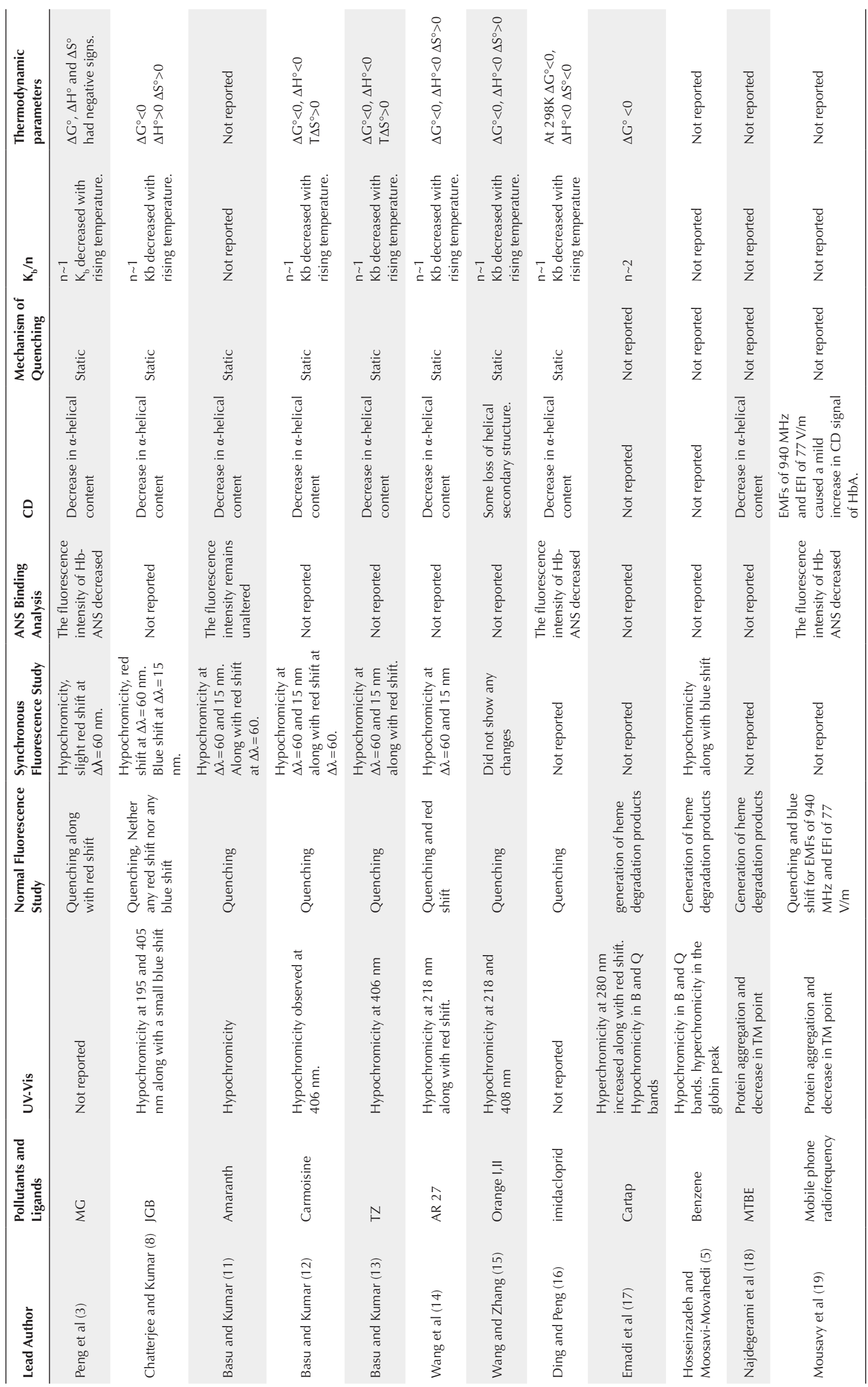




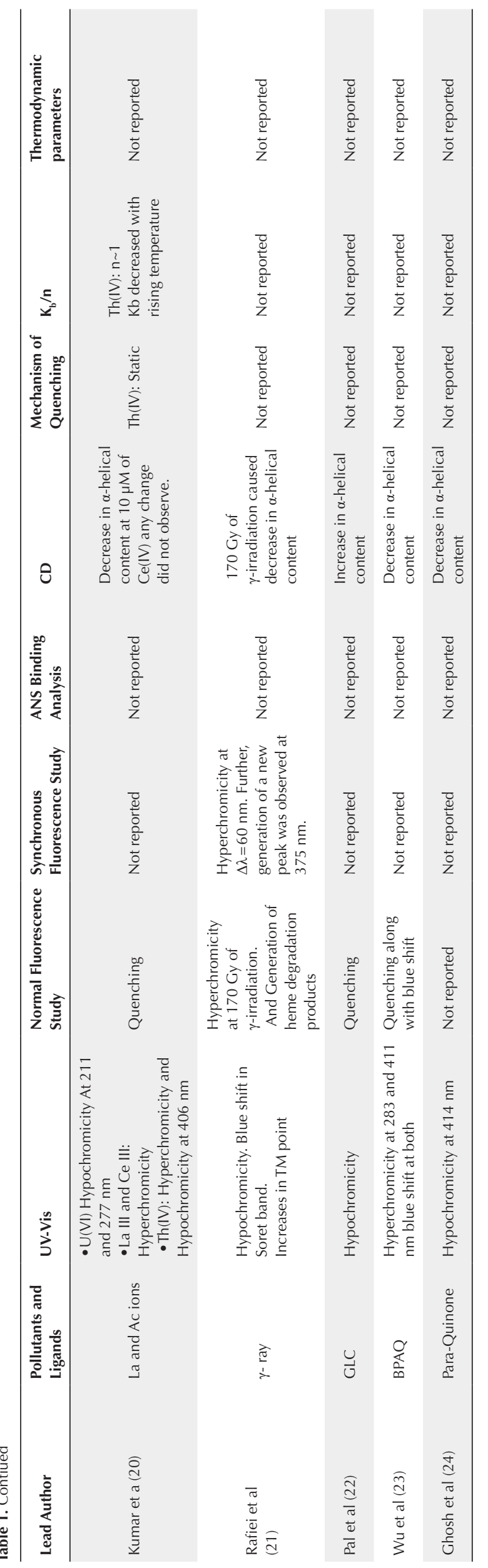


of these bands, suggesting the reduction of the oxy$\mathrm{Hb}$ and subsequently slowly expansion of deoxy and metHb (5). Under severe conditions, decrease in the total concentration of each three species of $\mathrm{Hb}$ (i.e., oxy, deoxy, and met) can be observed. In the case of $\gamma$-irradiated $\mathrm{HHb}$, hypochromicity and blue shift of the Soret band were in a good agreement with heme destruction phenomenon. Red shift that occurred at $348 \mathrm{~nm}$ upon $60 \mathrm{~Gy} \gamma$-irradiation, indicating the breaking of His-Fe (histidine-iron) bonds. Further, increasing intensity at $367 \mathrm{~nm}$ indicated the creation of some Fe-N (iron- nitrogen) weak bonds that resulted in the reduction of active oxy form and increment of inactive species such as deoxy- and metHb. Recent findings evidenced that there was a competitive behavior between benzene and oxygen for the oxy binding site. The affinity of benzene for this site was higher than that of oxygen, and that is why the addition of benzene to $\mathrm{Hb}$ solution decreased the total concentration of the protein. This finding was further supported by the data obtained from heme degradation study (26).

More investigations demonstrated that both MTBE and benzene mainly interact with hydrophobic amino acids in the hydrophobic pocket of $\mathrm{Hb}$, and due to such interaction, heme moiety is destroyed, and its degradation products could be detected through fluorimetry analysis. In the case of benzene, this phenomenon was further proved by reduction of $\mathrm{Hb}$ absorption at Soret bond, which resulted from penetration of benzene to the protein hydrophobic pocket and its direct interaction with the iron center (5). For $\gamma$-irradiation, it can be said that the peak intensities of the first group of heme degradation products, which also appeared after excitation at $321 \mathrm{~nm}$, increased 3 times after 170 Gy $\gamma$-irradiation. With regard to the second products that were also created after excitation at $460 \mathrm{~nm}$ due to ROS generation, peak intensities raised up by $90 \mathrm{~Gy}$ and then diminished by 170 Gy doses (21).

In addition to the above-mentioned observations, benzene could alter the protein conformational properties during interaction with aromatic residues and increase the hydrophobicity around them, which resulted in hyperchromicity and red shift in the globin absorption peak at $280 \mathrm{~nm}$. The conformational change in $\mathrm{Hb}$ led to loss of protein stability that resulted in reduction of protein's $\mathrm{Tm}$ point from $75.9^{\circ} \mathrm{C}$ to $74.2^{\circ} \mathrm{C}$ upon interaction with benzene and from $69^{\circ} \mathrm{C}$ to $67^{\circ} \mathrm{C}$ in 170 Gy $\gamma$-irradiated $\mathrm{Hb}$ samples, meaning that the unstable protein is degraded in lower temperature than stable one (5). Decline of the peak area in derivative plot $(\triangle \mathrm{A} 280$ versus $\Delta \mathrm{T}) / \mathrm{T}$ of $\gamma$-irradiated $\mathrm{Hb}$ samples indicated protein sedimentation or protein outgoing of solution. Further, generation of a new peak at $59^{\circ} \mathrm{C}$ demonstrated creation of less stable products.

Polarity alteration around aromatic residues was further supported by synchronous fluorimetry, and results indicated that both Tyr and Trp residues were involved in the interaction with benzene. Additionally, both Tyr and Trp peak intensities raised and shifted blue at $\Delta \lambda=17$ and $\Delta \lambda=80 \mathrm{~nm}$, respectively, due to the contribution of hydrophobic forces in complexation. This blue shift along with red shift observed in globin peak both indicated the involvement of hydrophobic forces in complex formation (5). Investigating the results of synchronous fluorimetry of $\gamma$-irradiated $\mathrm{Hb}$ samples suggested a noticeable hyperchromicity at $278 \mathrm{~nm}$ as well as the appearance of a new peak at $375 \mathrm{~nm}$ both at $\Delta \lambda=60 \mathrm{~nm}$ that belonged to $\operatorname{Trp}$ residues. The observed hyperchromicity and generation of the new peak at $375 \mathrm{~nm}$ illustrated the elimination of filtering heme group and creation of some heme degradation moieties, respectively (21).

It was demonstrated that with high concentrations of hemoglobin, the heme group acted as a quencher and induced about $99 \%$ of non-radiative quenching related to the intrinsic fluorescence of the aromatic fluorophores in some systems (5). Although heme is not a fluorescent compound, its degradation products are regarded as a fluorescent compound. Hence, after degradation of this group, its filtering effect disappears and causes hyperchromicity in intrinsic and even synchronous fluorescence spectra just like what have been observed in $\gamma$-irradiated $\mathrm{Hb}$ samples $(21,26)$. Fluorescence hyperchromicity is also considered as heme degradation indicator. In the case of benzene, both Uv-vis and far UV $\mathrm{CD}$ results were consistent with the results which obtained from P-benzoquinone study.

Of the seven studies reviewed on dyes, five studies were conducted on azo dyes, and two other studies investigated the impacts of a triaryl methane and phenazinium dyes on $\mathrm{Hb}$. Increasing the concentrations of each azo dyes except AR 27 contributed to a significant hypochromicity in the Soret band while the positions of peaks remained unaltered. Such spectral changes can be associated with an intimate contact between azo dyes and $\mathrm{Hb}(1,5,21)$, which could be more confirmed by the results of intrinsic spectrofluorimetry. In the case of JGB as a phenazinium dye, hypochromicity could be observed in both globin and Soret band of $\mathrm{Hb}$ absorbance spectrum, which is considered as formation of a ground state complex between the dye and $\mathrm{Hb}$ (27).

The findings demonstrated that all seven dyes potentially decreased the fluorescence of $\mathrm{Hb}$, and the nature of this quenching found to be static due to the complex formation between each dyes and $\mathrm{Hb}$. Since the intrinsic fluorescence of $\mathrm{Hb}$ was generally raised from the $\beta-37$ Trp residue at the $\alpha 1 \beta 2$ interface (28-30), any conformational change in the protein led to change in the fluorescence emission and polarity around $\beta-37$ Trp microenvironment.

Synchronous fluorescence analysis at $\Delta \lambda=60$ and 15 $\mathrm{nm}$, which belonged to Trp and Tyr residues, respectively, indicated that all seven dyes could alter the polarity around $\beta-37$ Trp and direct it to more polar environments. Among the studied dyes, only AR 27, Orange I and II, as well as JGB could induce transformation in the microenvironment of Tyr residues in such a way that two first dyes decreased hydrophobicity around them, and the 
last one exposed them to polar solvent. This demonstrated more involvement of Trp residue in Hb-azo dye complex formation.

The far-UV CD curve of hemoglobin exhibited two negative peaks in the far-UVCD region at $208 \mathrm{~nm}$ and $222 \mathrm{~nm}$, which refer to $\Pi \rightarrow \Pi^{*}$ transition of the $\alpha$-helix and $\mathrm{n} \rightarrow \Pi^{\star}$ transition for both the $\alpha$-helix and random coil, respectively (31). Upon binding to each seven dyes, the $\mathrm{CD}$ spectrum of $\mathrm{Hb}$ diminished in magnitude, suggesting the decrease in the helical content of $\mathrm{Hb}$. Accordingly, azo dyes induced unfolding and loss of a large part of the helical stability of $\mathrm{Hb}$, causing extended polypeptide chains to simultaneously expose the hydrophobic cavities to the aromatic amino acid residues. Additionally, interaction of MG with the amino acid residues of the polypeptide chain of $\mathrm{Hb}$ led to unfolding of the protein.

The decrement of the values of binding constant for MG, carmoisine, AR 27, JGB, and TZ along with increasing temperature indicated the destabilization of the hemoglobin-dye complexes at higher temperatures which was considered as evidence for complex formation and static quenching mechanism (12-14). In addition, the values of the number of binding sites were approximately equal to one for each seven dyes, suggesting the existence of only one kind of binding site in $\mathrm{Hb}$ for them. According to the ANS displacement studies, this binding site for amaranth is different from hemoglobin central cavity; therefore, amaranth binding reaction was not influenced by ANS-Hb complex formation. In contrast, upon addition to Hb-ANS mixture, the dye MG could compete with ANS probe for attachment to the $\mathrm{Hb}$ central cavity and dislocate the probe from that, which consequently weaken the extrinsic fluorescence intensity. As known, 1,8-anilinonaphthalenesulfonic (ANS) has high affinity for hydrophobic regions of the central cavity of $\mathrm{Hb}$; hence, any small molecules which is able to attach to these regions would compete with ANS, dislocate ANS, and replace it(32). Therefore, displacing ANS from its favorable binding site resulted in decrement of extrinsic fluorescence intensity. As noted earlier, the intrinsic fluorescence of $\mathrm{Hb}$ is mainly attributed to the $\beta-37 \operatorname{Trp}$ residue; therefore, the value of the number of binding sites indicated that the ligand binding site is moret likely near to this residue, causing fluorescence quenching. The negative values of $\Delta \mathrm{H}^{\circ}\left(-28.18 \mathrm{~kJ} \mathrm{~mol}^{-1}\right)$ and $\Delta \mathrm{S}^{\circ}(-14.92$ $\mathrm{J} \mathrm{mol}^{-1} \mathrm{~K}^{-1}$ ) attested that both hydrogen bonds and van der Waals forces play a key role in the case of the $\mathrm{Hb}-$ MG complexation mechanism. The negative signs of $\Delta \mathrm{G}^{\circ}$ and $\Delta \mathrm{H}^{\circ}$ and positive sign of $\Delta \mathrm{S}^{\circ}$ for interaction of $\mathrm{Hb}$ with AR 27 and Orange I and II demonstrated that the binding process was spontaneous, and electrostatic and hydrophobic interactions were involved in complexation process (33). The signs of thermodynamic parameters in the case of JGB-Hb interaction was obtained to be $\Delta \mathrm{G}^{\circ}<0$, $\Delta H^{\circ}>0$ and $\Delta S^{\circ}>0$, indicating the endothermic reaction and involvement of hydrogen bonds through interaction.

In a study conducted on the impacts of lanthanides and actinides on $\mathrm{Hb}$ properties, it was found that the lanthanides (La)s and actinides (Ac)s metal ions such as $\mathrm{Ce}(\mathrm{IV})$ and $\mathrm{Th}(\mathrm{IV})$, respectively, whose charge-toionic-radius ratio are equal to $\mathrm{Fe}(\mathrm{III})$, diminished heme absorbance significantly compared with actinide metal ions of lower charge-to-ionic-radius ratio, including $\mathrm{La}(\mathrm{III})$ and $\mathrm{Ce}(\mathrm{III})$. Furthermore, $\mathrm{Ce}(\mathrm{IV})$ whose chargeto-ionic-radius ratio is more closer to $\mathrm{Fe}(\mathrm{III})$ was found to be most effective in decreasing the heme absorbance (34). Both actinides $\mathrm{Th}(\mathrm{IV})$ and U(VI) potentially quenched the $\mathrm{Hb}$ fluorescence. Decreasing the fluorescence intensity indicates the increase of polarity around aromatic residues such as Trp and Tyr, meaning that this interaction led protein to unfolding states. Due to the decrease in values of the $\mathrm{K}_{\mathrm{sv}}$ and $\mathrm{K}_{\mathrm{q}}$ along with raising temperature, the nature of $\mathrm{Hb}$ fluorescence quenching found to be static because increasing the temperature resulted in increment of the molecular collisions in dynamic quenching; accordingly, the values of $K_{s v}$ and $K_{q}$ raised with temperature $(6,31)$. Unfolding of $\mathrm{Hb}$ as a result of interaction with $\mathrm{La}$ and Acs was more displayed by the far-UV CD results, which demonstrated that all these ionic metals caused loss of helicity in the protein and led that into structural instability.

Another study carried out on the cartap insecticide indicated that thisinsecticide could alter the conformational and functional properties of $\mathrm{Hb}$ and porphyrin. This could be due to the increase and red shift in intensity of absorption at $280 \mathrm{~nm}$ (refers to the globin peak) as well as the decrease in both Soret region and Q band. The red shift indicated that the hydrophobic forces contributed to $\mathrm{Hb}$-cartap interaction. Such interaction could disturb the environment of heme and disrupt the oxygen binding to $\mathrm{Hb}$. This could be realized from reduction of Soret and Q band absorptions (5). The ability of cartap in induction of ROS resulted in the generation of heme degradation products which represent a sensitive marker for indicating $\mathrm{Hb}$ oxidative stress under pathological conditions (26). Decrease of the protein's Tm in the presence of cartap revealed destabilization of the protein and its unfolding in lower temperatures compared with native proteins which are further confirmed by thermodynamic results. Moreover, analysis of $\mathrm{Hb}$ aggregation potential via cartap indicated that the initiation of thermal aggregation of protein could also happen in the vicinity of native protein by forming intermolecular interactions in specific regions without needing the whole set of unfolded proteins. According to the thermodynamic parameters, there were 2 binding sites for cartap on $\mathrm{Hb}$, which interact with the insecticide through hydrogen bonds and hydrophobic forces (17). Effect of imidacloprid insecticide on $\mathrm{HHb}$ spectral properties was investigated. Obtained results showed the ability of imidacloprid for quenching of intrinsic fluorescence intensity of $\mathrm{HHb}$, meaning that the insecticide can interact with protein and change its conformational properties. The nature of this quenching was found to be static. Further studies evidenced that 
the insecticide had the potential to decrease the $\alpha$-helical content of protein which resulted in the reduction of protein compression. It can also change the polarity around Trp and Tyr residues, leading to their greater exposure to solvent. Furthermore, extrinsic fluorescence intensity of Hb-ANS complex demonstrated a significant diminish in the presence of insecticide, suggesting that ANS has the potential to compete with imidacloprid for $\mathrm{HHb}$ hydrophobic patches. This finding elucidated that the insecticide interact with $\mathrm{HHb}$ via hydrophobic sites. The negative signs of thermodynamic parameters displayed that non-covalent forces were involved in complex formation between $\mathrm{HHb}$ and insecticide (1).

It was found that the peak at $406 \mathrm{~nm}$ also resulted from conformational homogeneity of iron atom in the heme plane $(35,36)$, thus through the interaction between $\mathrm{Hb}$ and GLC, the change in the absorption of this region indicated that GLC could induce conformational heterogeneity in the protein, leading to protein unfolding. Since $\mathrm{Hb}$ fluorescence emission also resulted from its three Trp residues, the quenching of emission spectrum can be considered as polarity alteration in Trp microenvironment in the presence of GLC. Increase in the CD signals at 190, 209 and $222 \mathrm{~nm}$ demonstrated faster adsorption rate of $\mathrm{Hb}$ in the presence of GLC and absorption of aromatic rings of GLC. The existence of $\mathrm{H}$-bonding between heme group and GLC molecule, which evidenced from docking analysis, confirmed the results obtained from UV-vis spectroscopy. As we know, the transition of $\Pi \rightarrow \Pi^{\star}$ of phenyl groups of aromatic amino acids and hematoporphyrin ring in $\mathrm{Hb}$ led to absorption spectra in the range of 250-300 nm and around $410 \mathrm{~nm}$, respectively. Any alteration in absorbance of these regions was an indicative of conformational change in $\mathrm{Hb}$ polypeptide chain, heme moiety, and polarity alteration around its aromatic amino acids (22).

In the case of BPAQ, based on the UV-Vis findings, we can conclude the dispersion of aromatic amino acids in the aqueous solution in the subsequence of $\mathrm{Hb}$ destruction along with exposure of heme moiety to the solvent. Through the decrement of $\mathrm{Hb}$ fluorescence intensity and reduction of the CD signals at two negative peaks of 209 and 222 $\mathrm{nm}$, it could be understood that BPAQ, despite complex formation with $\mathrm{Hb}$, could affect the protein secondary structure and reduce its helical stability and compactness (23). In the study performed on the $\mathrm{HbA}$ by exposure to EMFs, it was concluded that $940 \mathrm{MHz}$ of EMFs with 77 $\mathrm{V} / \mathrm{m}$ of EFI led to more compact structure of the protein. Accordingly, $2^{\circ} \mathrm{C}$ increment of Tm point accompanied by reduction of ANS signal of exposed $\mathrm{Hb}$ was considered as strong evidences for these findings compared with unexposed one. Further, the blue shift observed during fluorimetry analysis displayed movement of Trp residues to more hydrophobic places through exposure to the protein. Although the blue shift of emission maxima is always accompanied by increase in fluorescence intensity, sometimes due to the high concentrations of HbA solution, the amount of heme moiety raises and quenches the Trp fluorescence. Faster reaching of exposed $\mathrm{HbA}$ to the plateau state compared with unexposed one after 175 minutes means that the EMFs induced less hydrophobic patches on the $\mathrm{HbA}$ surface, and it caused rapid sedimentation of the exposed protein in the solvent. From the CD analysis, it can be concluded that exposure of HbA to EMFs did not affect the secondary structure but led to the reduction of the Soret CD spectra, which raises from $\Pi \rightarrow \Pi^{\star}$ transition of the heme chromophore, meaning that exposure to the radiation affected globin-heme interaction (19). Figure 2 briefly displays the main mechanism of interaction between $\mathrm{HHb}$ and toxic exogenous ligands.

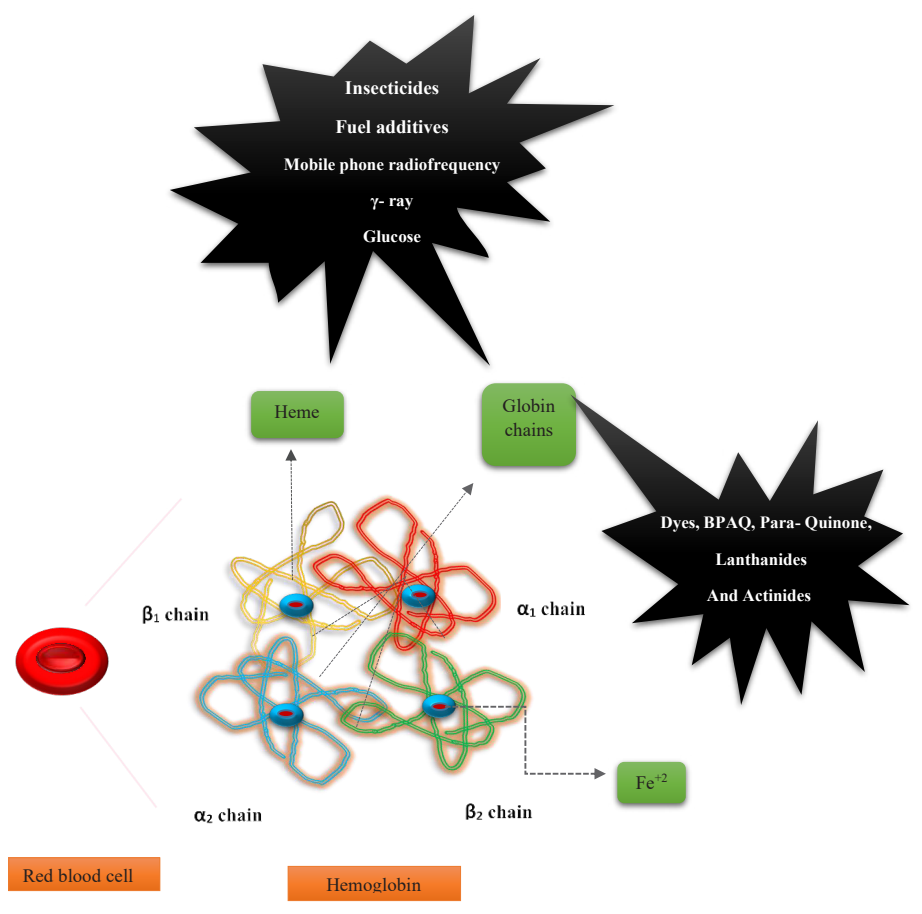

Figure 2. The scheme of interaction between exogenous toxic ligands and different parts of HHb. Note. HHb: Human hemoglobin. 


\section{Conclusion}

The findings of the present study demonstrated that many synthetic compounds which we consume in our daily life, despite their harmful effects on environment and living organisms, can also endanger our life. In addition to agents that have added to foodstuffs, the risk of exposure to environmental pollutants cannot be ignored. As we described in our review, exposure to these compounds caused conformational changes in $\mathrm{HHb}$. Higher affinity of some of these compounds to penetrate the hydrophobic pocket resulted in heme destruction and elimination of heme filtering group. Furthermore, competition between $\mathrm{HHb}$ and some pollutants for attachment to oxygen binding site reduced the oxy form of $\mathrm{HHb}$ which resulted in irreparable damages to human health and other living organisms. To overcome these problems, in the first place, governments must monitor the production and commercialization of chemical products, and secondly, industry owners have to focus on the functions and effects of chemical products on human and living organisms before commercializing theme.

Conflict of interests

There is no conflict of interests regarding publication of this article.

\section{Ethical Considerations}

In writing the article, the principles of publishing ethics have been observed.

\section{Authors' Contributions}

AKS edited the article and proposal and ERP performed the data collection and wrote the article.

\section{References}

1. Ding F, Han BY, Liu W, Zhang L, Sun Y. Interaction of imidacloprid with hemoglobin by fluorescence and circular dichroism. J Fluoresc. 2010;20(3):753-62. doi: 10.1007/ s10895-010-0618-0.

2. Liu W, Guo X, Guo R. The interaction between hemoglobin and two surfactants with different charges. Int J Biol Macromol. 2007;41(5):548-57. doi: 10.1016/j.ijbiomac.2007.07.006.

3. Peng W, Ding F, Peng YK, Sun Y. Molecular recognition of malachite green by hemoglobin and their specific interactions: insights from in silico docking and molecular spectroscopy. Mol Biosyst. 2014;10(1):138-48. doi: 10.1039/c3mb70416e.

4. Wang YQ, Zhang HM, Zhang GC, Liu SX, Zhou QH, Fei ZH, et al. Studies of the interaction between paraquat and bovine hemoglobin. Int J Biol Macromol. 2007;41(3):243-50. doi: 10.1016/j.ijbiomac.2007.02.011.

5. Hosseinzadeh R, Moosavi-Movahedi AA. Human hemoglobin structural and functional alterations and heme degradation upon interaction with benzene: a spectroscopic study. Spectrochim Acta A Mol Biomol Spectrosc. 2016;157:41-9. doi: 10.1016/j.saa.2015.12.014.

6. Shanmugaraj K, Anandakumar S, Ilanchelian M. Unraveling the binding interaction of Toluidine blue $\mathrm{O}$ with bovine hemoglobin-a multi spectroscopic and molecular modeling approach. RSC Adv. 2015;5(6):3930-40. doi: 10.1039/ c4ra11136b.

7. Valipour M, Maghami P, Habibi-Rezaei M, Sadeghpour M, Khademian MA, Mosavi K, et al. Counteraction of the deleterious effects of reactive oxygen species on hemoglobin structure and function by ellagic acid. J Lumin. 2017;182:1-7. doi: 10.1016/j.jlumin.2016.10.003.
8. Chatterjee S, Kumar GS. Targeting the heme proteins hemoglobin and myoglobin by janus green blue and study of the dye-protein association by spectroscopy and calorimetry. RSC Adv. 2014;4(80):42706-15. doi: 10.1039/c4ra06600f.

9. Divito CB, Davies S, Masoudi S, Muhoro CN. Relative stability of formamidine and carbamate groups in the bifunctional pesticide formetanate hydrochloride. J Agric Food Chem. 2007;55(14):5377-82. doi: 10.1021/jf0637527.

10. Cui Y, Guo J, Xu B, Chen Z. Binding of chlorpyrifos and cypermethrin to blood proteins. Pestic Biochem Physiol. 2006;85(2):110-4. doi: 10.1016/j.pestbp.2005.11.004.

11. Basu A, Kumar GS. Interaction of toxic azo dyes with heme protein: biophysical insights into the binding aspect of the food additive amaranth with human hemoglobin. J Hazard Mater. 2015;289:204-9. doi: 10.1016/j.jhazmat.2015.02.044.

12. Basu A, Suresh Kumar G. Binding of carmoisine, a food colorant, with hemoglobin: spectroscopic and calorimetric studies. Food Res Int. 2015;72:54-61. doi: 10.1016/j. foodres.2015.03.015.

13. Basu A, Suresh Kumar G. Multispectroscopic and calorimetric studies on the binding of the food colorant tartrazine with human hemoglobin. J Hazard Mater. 2016;318:468-76. doi: 10.1016/j.jhazmat.2016.07.023.

14. Wang YQ, Zhang HM, Tang BP. The interaction of C.I. acid red 27 with human hemoglobin in solution. J Photochem Photobiol B. 2010;100(2):76-83. doi: 10.1016/j.jphotobiol.2010.05.004.

15. Wang YQ, Zhang HM. Investigations on the binding of human hemoglobin with orange I and orange II. J Photochem Photobiol B. 2012;113:14-21. doi: 10.1016/j.jphotobiol.2012.04.009.

16. Ding F, Peng W. Biological assessment of neonicotinoids imidacloprid and its major metabolites for potentially human health using globular proteins as a model. J Photochem Photobiol B. 2015;147:24-36. doi: 10.1016/j. jphotobiol.2015.03.010.

17. Emadi M, Maghami P, Khorsandi K, Hosseinzadeh R. Biophysical study on the interaction of cartap hydrochloride and hemoglobin: heme degradation and functional changes of protein. J Biochem Mol Toxicol. 2019;33(7):e22325. doi: 10.1002/jbt.22325.

18. Najdegerami IH, Maghami P, Sheikh-Hasani V, Hosseinzadeh G, Sheibani N, Moosavi-Movahedi AA. Antichaperone activity and heme degradation effect of methyl tert-butyl ether (MTBE) on normal and diabetic hemoglobins. J Mol Recognit. 2017;30(5):10.1002/jmr.2596. doi: 10.1002/jmr.2596.

19. Mousavy SJ, Riazi GH, Kamarei M, Aliakbarian $H$, Sattarahmady N, Sharifizadeh A, et al. Effects of mobile phone radiofrequency on the structure and function of the normal human hemoglobin. Int J Biol Macromol. 2009;44(3):278-85. doi: 10.1016/j.ijbiomac.2009.01.001.

20. Kumar A, Ali M, Ningthoujam RS, Gaikwad P, Kumar M, Nath $\mathrm{BB}$, et al. The interaction of actinide and lanthanide ions with hemoglobin and its relevance to human and environmental toxicology. J Hazard Mater. 2016;307:281-93. doi: 10.1016/j. jhazmat.2015.12.029.

21. Rafiei J, Yavari K, Moosavi-Movahedi AA. Preferential role of iron in heme degradation of hemoglobin upon gamma irradiation. Int J Biol Macromol. 2017;103:1087-95. doi: 10.1016/j.ijbiomac.2017.05.153.

22. Pal P, Mahato M, Kamilya T, Talapatra GB. Interaction of glucose with hemoglobin: a study in aqueous solution and at the air-water interface using the Langmuir-Blodgett technique. Phys Chem Chem Phys. 2011;13(20):9385-96. doi: 10.1039/ c0cp02277b.

23. Wu Q, Zhao H, Chen X, Cai Z. Interaction of bisphenol A 3, 4-quinone metabolite with human hemoglobin, human serum albumin and cytochrome $\mathrm{c}$ in vitro. Chemosphere. 2019;220:930-6. doi: 10.1016/j.chemosphere.2018.12.194.

24. Ghosh A, Banerjee S, Mitra A, Muralidharan M, Roy B, 
Banerjee $\mathrm{R}$, et al. Interaction of p-benzoquinone with hemoglobin in smoker's blood causes alteration of structure and loss of oxygen binding capacity. Toxicol Rep. 2016;3:295305. doi: 10.1016/j.toxrep.2016.02.001.

25. Khan MS, Tabrez S, Bhat SA, Rabbani N, Al-Senaidy AM, Bano B. Effect of trifluoroethanol on a-crystallin: folding, aggregation, amyloid, and cytotoxicity analysis. J Mol Recognit. 2016;29(1):33-40. doi: 10.1002/jmr.2493.

26. Nagababu E, Rifkind JM. Formation of fluorescent heme degradation products during the oxidation of hemoglobin by hydrogen peroxide. Biochem Biophys Res Commun. 1998;247(3):592-6. doi: 10.1006/bbrc.1998.8846.

27. Kay RE, Walwick ER, Gifford CK. Spectral changes in a cationic dye due to interaction with macromolecules. II. Effects of environment and macromolecule structure. J Phys Chem. 1964;68(7):1907-16. doi: 10.1021/j100789a041.

28. Fang X, Cao S, Liu R. Interaction of bisphenol A with bovine hemoglobin using spectroscopic and molecular modeling methods. Appl Spectrosc. 2011;65(11):1250-3. doi: 10.1366/11-06357.

29. Maity S, Chakraborty S, Chakraborti AS. Critical insight into the interaction of naringenin with human haemoglobin: a combined spectroscopic and computational modeling approaches. J Mol Struct. 2017;1129:256-62. doi: 10.1016/j. molstruc.2016.09.085.

30. Wang L, Liu R, Chi Z, Yang B, Zhang P, Wang M. Spectroscopic investigation on the toxic interactions of $\mathrm{Ni} 2+$ with bovine hemoglobin. Spectrochim Acta A Mol Biomol Spectrosc. 2010;76(2):155-60. doi: 10.1016/j.saa.2010.03.006.

31. Xiao M, Yuan X, Xie W, Ge X, Zhou Y, Zhou L, et al. Comparison of 9-hydroxy-artemisinin with artemisinin: interaction with bovine hemoglobin. J Lumin. 2015;160:18894. doi: 10.1016/j.jlumin.2014.12.002.

32. Syakhovich VE, Parul DA, Ruta EY, Bushuk BA, Bokut SB. 1,8-Anilinonaphthalene sulfonate binds to central cavity of human hemoglobin. Biochem Biophys Res Commun. 2004;317(3):761-7. doi: 10.1016/j.bbrc.2004.03.111.

33. Liu W, Ding F, Sun Y. Characterization of phenosafraninehemoglobin interactions in aqueous solution. J Solution Chem. 2011;40(2):231-46. doi: 10.1007/s10953-010-9647-1.

34. Hartman P, Chan HK. Application of the periodic bond chain (PBC) theory and attachment energy consideration to derive the crystal morphology of hexamethylmelamine. Pharm Res. 1993;10(7):1052-8. doi: 10.1023/a:1018927109487.

35. Mahato M, Pal P, Kamilya T, Sarkar R, Chaudhuri A, Talapatra GB. Hemoglobin-silver interaction and bioconjugate formation: a spectroscopic study. J Phys Chem B. 2010;114(20):7062-70. doi: 10.1021/jp100188s.

36. Boffi A, Verzili D, Chiancone E, Leone M, Cupane A, Militello $\mathrm{V}$, et al. Stereodynamic properties of the cooperative homodimeric Scapharca inaequivalvis hemoglobin studied through optical absorption spectroscopy and ligand rebinding kinetics. Biophys J. 1994;67(4):1713-23. doi: 10.1016/s00063495(94)80645-9. 